Programmatische Kommentare und Repliken

Susanne Leeb* und Ruth Sonderegger**

\title{
Plädoyer für eine kulturwissenschaftliche Ästhetik aus Perspektive der cultural studies
}


Abstract: Our comment on Hartmut Böhme advocates an approach to aesthetics that is mainly inspired by British cultural studies. In the wake of the foundation of the "Kulturwissenschaftliche Gesellschaft" and its journal we suggest, on the one hand, a relentless reflection on essentialist and colonialist power structures inherent in the concept of culture, particularly in the German speaking world. On the other hand, we plea for the provincialization of European aesthetics as well as for the acknowledgement of the manifold entanglements between European and non-European accounts of aesthetics.

Keywords: cultural studies, dekoloniale Ästhetiken, decolonial aesthetics, entangled histories, essentialistische und/oder rassifizierende Kulturbegriffe, Eurozentrismus der Ästhetik, high/low, Machtkritik, postcolonial studies, Provinzialisierung Europas, self-othering, critique of power

\footnotetext{
*Prof. Dr. Susanne Leeb: Institut für Philosophie und Kulturwissenschaft, Leuphana Universität Lüneburg, Scharnhorststraße 1, C5.410, D-21335 Lüneburg, email: susanne.leeb@leuphana.de

**Prof. Dr. Ruth Sonderegger: Institut für Kunst- und Kulturwissenschaften, Akademie der bildenden Künste Wien, Schillerplatz 3, A-1010 Wien, email: r.sonderegger@akbild.ac.at
}

\section{Kritik an kolonialen Kulturbegriffen}

Wenn zur Moderne ebenso wie zu den Kulturwissenschaften eine Reflexivitätszunahme gehört, wie Hartmut Böhme im ersten Absatz seiner Überlegungen schreibt, dann stehen so zentrale Begriffe wie "Moderne" und "Kultur" ebenfalls zur Disposition - insbesondere in historischer und geopolitischer Hinsicht. Nach den vielfältigen von anti-, post- und dekolonialen Ansätzen formulierten Einwänden gegen die Setzung europäischer Modernitätsansprüche als der Moderne schlechthin, hat dieser Begriff jeden Anschein von Selbstverständlichkeit verloren. ${ }^{1}$ Ähnliches

1 Vgl. dazu für das Feld der Künste z.B. Mercer (2005) und Avermaete/Karakayali/von Osten (2010). Vor diesem Hintergrund fällt es uns schwer, die hervorgehobene Rolle Europas in den Überlegungen von Böhme nachzuvollziehen, etwa wenn er schreibt: „Eine historische Kulturwissenschaft versteht ihre Rolle vor allem darin, die langwellige Entstehung europäischer Kulturen, aber auch ihres Zugs zur Globalisierung zu untersuchen. [...] Insbesondere erweisen sich die Künste und die Techniken als Motoren übernationaler, ,europäischer' Praktiken und kultureller Leitbilder. In dieser longue durée ist Kulturwissenschaft zu situieren." (vgl. Böhme in diesem Heft) Gegen diese Vorstellung, wonach der Fortschritt von Europa aus in die Welt hinaus getragen wird, hat beispielsweise Di- gilt für den Begriff der Kultur, zumal in seiner deutschsprachigen Herkunft. Gerade in einem Gründungsmoment - desjenigen der Kulturwissenschaftlichen Gesellschaft und ihrer Zeitschrift - steht die Frage im Raum, worauf die Gründung sich gründet. Dass die deutschsprachige Tradition der Kulturwissenschaften des 19. Jahrhunderts Rasseanthropologien und essentialistische wie kolonialistische Kulturbegriffe mitgetragen hat, sollte im Moment des postulierten Anfangs oder Neuanfangs ebenso Thema sein wie die Rolle des deutschen Kulturbegriffs im Nationalsozialismus. ${ }^{2}$

Wenn es bei Böhme unter dem Stichwort "Entwicklungen der Moderne in den letzten 250 Jahren" nicht zuletzt um die „Funktionen der sog. freien und der nützlichen Künste" (vgl. Böhme in diesem Heft) geht, verstehen wir die Hinterfragung dieser modernen Dichotomie als eine Möglichkeit, die verpflichtet - unter anderem dazu zu erkennen, dass die okzidentale Moderne im Hin-

pesh Chakrabarty (2007) überzeugend eine notwendige Provinzialisierung Europas stark gemacht. Theoretiker_ innen wie Valentin Mudimbe oder Gayatry Chakravorty Spivak haben gegen die genannte Vorstellung schon seit langem protestiert. Das Universalismusproblem, an dem solche Autor_innen arbeiten, wäre auch für die Ästhetikdiskussion produktiv zu machen.

2 Instruktiv für v.a. das 19. Jahrhundert ist in dieser Hinsicht beispielsweise Smith (1991). 
blick auf ihre eigenen Prämissen nicht einmal halb so reflexiv ist, wie sie gerne von sich behauptet. Wir möchten daher skizzieren, mit welchen weiteren Fragen eine Ästhetik aus kulturwissenschaftlicher Perspektive noch befasst sein sollte.

\section{Kritik an der Macht der Ästhetik}

Eine im Vergleich mit den deutschen Kulturwissenschaften weitaus (selbst-)kritischere Auseinandersetzung mit den eigenen Grundbegriffen zeichnet die Birminghamer Schule der Cultural Studies aus, wie sie am Centre for Contemporary Cultural Studies (CCCS) entwickelt worden sind (vgl. Sonderegger 2004). Das ist nicht zuletzt der Überzeugung der Vertreter_innen dieser Schule geschuldet, wonach Kulturwissenschaften zu betreiben nicht bedeutet, mehr oder weniger positivistisch einer Wissenschaft nachzugehen, sondern (Theorie-)Politik zu machen und diese im Anschluss an Gramsci und Foucault - in ihren Macht-Effekten immer wieder neu zu verantworten bzw. verändernd in Geschichtsschreibung und Gegenwart einzugreifen. ${ }^{3}$ In diesem Sinne verstehen ihre Vertreter_innen auch den Kulturbegriff: „[...] cultural studies is both an intellectual and a political tradition. There is a kind of double articulation of culture in cultural studies, where ,culture' is simultaneously the ground on which analysis proceeds, the object of study, and the site of political critique and intervention." (Nelson et al. 1992: 5) Obwohl Machtanalysen im Zentrum der Arbeit am CCCS standen, ist die

3 Diesem Verständnis von Kulturwissenschaft folgend schreibt etwa Diedrich Diederichsen (1996), S. 54f.: "Diese Wissenschaftler [= Vertreter_innen der britischen Cultural Studies] sind stark in allgemeinen gesellschaftlichen Auseinandersetzungen eingebunden, veröffentlichen auch in populären Medien und verbinden so den schon von Raymond Williams in seinen TV-Kolumnen erhobenen Anspruch, die besagten ,Kulturen' nicht nur zu untersuchen, sondern, gut gramscianisch, sich als Aktivisten im Kampf um ihre Besetzung zu verstehen [...]." Dass Diederichsens Überlegungen in der Spex publiziert wurden, ist kein Zufall. Diese Zeitschrift war eine der entscheidenden Instanzen in Sachen deutschsprachige Diskussion und Rezeption der britischen Cultural Studies, neben einzelnen frühen Aufsätzen wie etwa in Ästhetik und Kommunikation 24 (1976) mit dem Themenschwerpunkt "Freizeit im Arbeiterviertel". Darüber hinaus hatte Diederichsen 1983 Dick Hebdiges Buch Subculture: The Meaning of Style (1976) übersetzt.
Zirkulation von ästhetischen Zeichen den Cultural Studies zufolge jedoch kein panoptisches Gefängnis. Vielmehr geht es darum, die Möglichkeiten der Veränderung - der Um- und Neukodierung, wie Stuart Hall damals sagte - zu finden (vgl. Hall 1980). Es ist kein Zufall, dass die meisten Vertreter_innen der frühen Birminghamer Cultural Studies aus der Erwachsenenbildung oder aus dem Kultur-Journalismus kamen und über diese Tätigkeiten für und mit Bevölkerungsgruppen, die aufgrund von klassistischen und rassialisierenden Strukturen ausgegrenzt waren, eine Perspektive im Großbritannien der 1960er, 70er und 80er Jahre verschaffen wollten. ${ }^{4}$ Dieser Zugang scheint uns auch heute noch in Bezug auf eine kulturwissenschaftliche Perspektive auf Fragen der Ästhetik und der Künste fruchtbar. ${ }^{5}$

Dabei geht es nicht nur darum, die populärkulturellen Anteile hervorzuheben. Vielmehr besteht auch aus der Perspektive der heutigen Cultural Studies ein wesentlicher Aspekt der Beschäftigung mit (philosophischer) Ästhetik, Kunsttheorie, Kunstgeschichte und Kunst darin, die Politiken sowie die intendierten und nicht-intendierten Machteffekte spezifischer Einsätze der Begriffe Kunst, Kultur und Ästhetik zu analysieren - nicht zuletzt auch die der jeweils eigenen Einsätze. ${ }^{6}$ Stellt man diese Machtfrage ins Zentrum, dann kann man sich nicht damit begnügen, die Theorien ästhetischer Autonomie mit Rousseau bzw. zur Zeit der Aufklärung beginnen zu lassen und darauf hinzuweisen, dass mit der Erfindung der

4 Böhme weist zu Recht darauf hin, dass die Cultural Studies "ohne den politischen Hintergrund der proletarischen Kultur nicht verständlich wären" (vgl. Böhme, ebd.). Insbesondere für die britischen Cultural Studies sind die antiund postkoloniale sowie die migrantische Kultur allerdings ebenso wichtig. Zum Zusammenhang der proletarischen mit der postkolonialen und der migrantischen Kultur im England der 1970er Jahre vgl. Hall (1978).

$5 \mathrm{Zu}$ den Bezugnahmen der deutschsprachigen Kulturwissenschaften auf die Britischen Cultural Studies vgl. beispielsweise Ege (2014). Zur Rezeption in den 1990er Jahren in den kulturwissenschaftlichen Studiengängen vgl. Bromley et al. (1999); vgl. auch die Studiengangvorstellung von Wuggenig (2001). Zur deutschsprachigen Rezeption allgemein vgl. Engelmann (1999); Hepp (1999); Hörning/Winter (1999).

6 Vgl. etwa Hall/Evans/Nixon (22013). Zu Halls Engagement für Black Art, das schließlich zur Gründung des Institute of International Visual Art (iniva) in London führte vgl. das BBC-Interview mit Hall auf: https://www.youtube. com/watch?v=GK3PX_e44z8. 
freien, selbstgenügsamen Künste auch die nützlichen nicht verschwinden, was zweifelsohne zutrifft (vgl. Böhme, ebd.). So sehr es stimmen mag, dass das Rousseau'sche Motiv des Ästhetischen als eines selbstgenügsamen und erfüllten Augenblicks von Schiller bis zu Adorno fortwirkt und sich gegen das Faustische Prinzip des "rastlos Schaffenden" (vgl. Böhme, ebd.) richtet, das der beginnende Industriekapitalismus zunehmend erbarmungslos einfordert, so sehr verlangte der Industriekapitalismus dies jedoch nicht von allen Menschen oder auch nicht nur von allen Europäer_innen gleichermaßen. ${ }^{7}$

Die Setzung selbstgenügsamer ästhetischer Augenblicke hat nicht nur Folgen jenseits der Linie von Schiller zu Adorno - und vielleicht ist sie viel weniger Setzung und geniale Erfindung als häufig behauptet. Es gibt auch Gründe, sie als ReAktion auf imperiale und koloniale Verhältnisse zu verstehen. Schon der Ethnologe Karl-Heinz Kohl (1981) und in jüngerer Zeit die Philosophin Iris Därmann (2005) haben dies für die Philosophie und einzelne Philosophen ebenso gezeigt, wie jüngst der Anglist Simon Gikandi mit seiner Darlegung der Verflochtenheit der Geschichte von der Kultivierung des Geschmacks mit der Geschichte der Sklaverei - eine doppelbödige Geschichte, die für die Gründungsperiode der englischen Ästhetik sowie der Institutionen, die daraufhin entstehen, ausschlaggebend ist. ${ }^{8}$ Die selbstgenügsamen Augenblicke der Ästhetik, die man mit Böhme aus der Geschichte der Arbeit und aus industriekapitalistischer Produktion herleiten kann, sind ohne handelsimperiale und koloniale Privilegien resp. Ausbeutungsverhältnisse nicht denkbar.

7 Zu einer internationaleren Kontextualisierung Rousseaus vgl. Därmann (2009), auf S. 73 mit der folgenden Analyse: "Zwar liefern Locke und Rousseau die entscheidenden aufgeklärten Prinzipien für die politisch-rechtliche Verurteilung des Kolonialismus und die gesetzliche Abschaffung des transatlantischen Sklavenhandels. Doch dienen namentlich ihre Naturzustandskonstruktionen dazu, die Universalität dieser Prinzipien mithilfe einer neuen Arbeitswertlehre zugleich lokal zu begrenzen und zwischen verschiedenen Rechts- und Raumzonen, zwischen den europäischen Nationen einerseits und ihren kolonialen Trabanten Amerika und Afrika andererseits aufzuteilen."

8 Gikandi (2011). Hier ließe sich Edward Saids "contrapuntal reading" anführen, wie er es in seinem Buch Culture and Imperialism (1993) entwickelt hat.

\section{Kritik am Eurozentrismus der Ästhetik}

Auch in deutschsprachigen Gründungsdokumenten des selbstgenügsamen ästhetischen Augenblicks wird die Trennung zwischen der freien und der nicht so freien Kunst auf dem Rücken des Fremden und Nicht-Männlichen ausgetragen. Nicht nur Kants frühe Schrift Beobachtungen über das Gefühl des Schönen und Erhabenen (1764) bringt das beklemmend krass zum Ausdruck. ${ }^{9}$ Auch am Beginn der Kritik der Urteilskraft, wo die reine ästhetische Lust von der unreinen des Geschmacks mit aller Gewalt geschieden wird, ist es der "irokesische Sachem", der die Primitivität des unreinen Geschmacks der Gaumenfreuden verkörpern und erklären muss. Denn Kant zufolge hat der irokesische Sachem keinen Sinn für die reine Schönheit von Paris, sondern nur für die "Garküchen" dieser Stadt (Kant: 1974, § 2). Diese Zuschreibung erklärt das europäische Subjekt zum normativen Ursprung der wahrhaft ästhetischen Erfahrung. Dagegen weist bell hooks - neben vielen anderen - mit Recht darauf hin: "It is really important to dispel the notion that white western culture is , the' location where a discussion of aesthetics emerged [...]; it is only one location". ${ }^{10}$

9 "Die $N^{*}$ [* von S.L., R.S.] von Afrika haben von der Natur kein Gefühl, welches über das Läppische stiege. Herr Hume fodert [sic!] jedermann auf, ein einziges Beispiel anzuführen, da ein $\mathrm{N}^{*}$ Talente gewiesen habe, und behauptet: daß unter Hunderttausenden von Schwarzen, die aus ihren Ländern anderwärts verführt werden, obgleich deren sehr viele auch in Freiheit gesetzt werden, dennoch nicht ein einziger jemals gefunden worden, der entweder in Kunst oder Wissenschaft, oder irgend einer andern rühmlichen Eigenschaft etwas Großes vorgestellt habe, obgleich unter den Weißen sich beständig welche aus dem niedrigsten Pöbel empor schwingen und durch vorzügliche Gaben in der Welt ein Ansehen erwerben. [...] Die unter innen weit ausgebreitete Religion der Fetische ist vielleicht eine Art von Götzendienst, welcher so tief ins Läppische sinkt, als es nur immer von der menschlichen Natur möglich zu sein scheinet. Eine Vogelfeder, ein Kuhhorn, eine Muschel, oder jede andere gemeine Sache, so bald sie durch einige Worte eingeweihet worden, ist ein Gegenstand der Verehrung und der Anrufung in Eidschwüren. Die Schwarzen sind sehr eitel, aber auf $\mathrm{N}^{*}$ art, und so plauderhaft, daß sie mit Prügeln müssen auseinander gejagt werden." In: Kant (1993), S.70f.

10 hooks (2015), S.103-113. Dieser Aufsatz ist nicht zuletzt deshalb instruktiv, weil bell hooks sich für die Trenn- 
Schließlich müsste die ästhetische Tradition, die in den Augen von Böhme von Schiller zu Adorno führt, auch dahingehend kontextualisiert (und ,provinzialisiert') werden, dass sie mit ästhetischen Theorien und Praktiken konfrontiert wird, die sich nicht von der, selbstredend auch in der europäischen Kunst und Kunsttheorie immer wieder umstrittenen Trennlinie zwischen freien und nützlichen, hohen und niedrigen Künsten her verstehen. Polynesische Tätowierungen etwa wurden der europäischen Kategorie des zweckIosen Ornaments subsumiert, obwohl sie soziale Marker und Individualitätszeichen sind ( $\mathrm{vgl}$. Leeb 2015). Gemäß dem marokkanischen Dichter Abdelatif Laabi, Mitbegründer der Zeitschrift Souffles, die in den 1960er Jahren in Marokko erschien, war die Trennung in "High" und "Low" eine Spaltung, gegen die anzuarbeiten zur wichtigsten Herausforderung für (in seinem Fall) nordafrikanische Künstler_innen und Intellektuelle der Dekolonialisierung geriet, um einen folkloristischen bis paternalistischen Blick auf lokale Kulturproduktion zurückzuweisen (vgl. von Osten/ Maharaj 2013: 141).

Mit einer kulturwissenschaftlichen Perspektive steht auch zur Debatte, welche Vorstellungen von Sinnlichkeit dem ästhetischen Urteil zugrunde liegen und in welcher Weise die okzidentale Rede von sinnlicher Erkenntnis einer sehr westlichen Geist/Körper-Dichotomie geschuldet ist. Weiterhin ist zu fragen, auf welchen normativen Vorstellungen diese Sinnlichkeit basiert und etwa die Privilegierung des Blicks zu problematisieren, von der der amerikanische Kunst-, Medien- und Kommunikationswissenschaftler Nicolas Mirzoeff schreibt: "all visuality was and is imperial visuality, the shaping of modernity from the point of view of the imperial powers." (Mirzoeff 2011: 196) Weiterhin liegt es angesichts des Wissens um die Verknüpfung von Rassenlehre und Ästhetik im 18. Jahrhundert und der Tatsache, dass gerade die Kategorie der Schönheit an moralische Überlegenheitsvorstellungen geknüpft war (vgl. etwa Lettow (2010); Bernasconi 2001), nahe, eine Analytik der Macht in Bezug auf die Kategorie der Schönheit vorzunehmen. Ebenso ist zu untersuchen, inwieweit die ästhetische Kategorie des Erhabenen, die Entdeckung des,Dunklen' im

linie zwischen den freien und den nützlichen Künsten offensiv nicht interessiert.
18. Jahrhundert nicht nur von einem sich zerteilenden und zerteilten Subjekt zeugen, sondern auch von Kolonialgeschichte. So hat die Hispanistin und Komparatistin Lizabeth Paravisini-Gebert in "Colonial and Postcolonial Gothic" betont, dass die Schauerliteratur seit ihren Anfängen im England und Frankreich des späten 18. Jahrhunderts ganz wesentlich an koloniale Schauplätze, Charaktere und Realitäten gebunden war: "With the inclusion of the colonial", so Paravisini-Gebert (2002: 229), "a new sort of darkness - of race, landscape, erotic desire and despair - enters the Gothic genre [...]." 11 Sie hebt auch ganz konkrete Verstrickungen hervor: So war William Beckford - Autor von Vathek, einem orientalistischen Schauerroman von 1786 - der Erbe eines enormen Vermögens aus drei Generationen Zuckerplantagenbesitzern in Jamaica und vertrat die Interessen von Sklavenbesitzern auf den Westindies im britischen Parlament (vgl. Paravisini-Gebert 2012: 230). Auch wenn Autor und Erzählung resp. ästhetische Strategien immer zu trennen sind, wäre hier im Sinne eines kulturellen Unbewussten zu untersuchen, inwieweit die Beteiligung am Sklavenhandel und am kolonialen Unterfangen sich in Ästhetiken der Gothic Novel niedergeschlagen hat.

Es wäre aus kulturwissenschaftlicher Perspektive überdies zu fragen, ob auch die Ästhetik als Phänomenologie ein eurozentrisches Subjekt privilegiert. Denn was bedeutet es für MerleauPontys Philosophie, dass er sich für Paul Gauguin interessierte, der sich ein Südseeideal zu kolonialen Bedingungen schuf? ${ }^{12}$ Wie ist ,der Mensch' verfasst, auf dem eine phänomenologische Ästhetik basiert? Inwieweit beinhaltet etwa MerleauPontys Ansicht, wir alle seien Invaginationen desselben Fleisches, welches nicht als Materie oder Geist zu denken sei, sondern als Stoff, der sich in allen Dingen und Körpern manifestiert, noch ein eurozentrisches Subjekt? Welches Subjekt- und Ontologieverständnis liegt demgegenüber einer relationalen Ästhetik zugrunde, wie sie etwa der

11 Die Schauerliteratur war ",[...] fundamentally linked to colonial settings, characters, and realities as frequent embodiments of the forbidding and frightening [...]. By the 1790s Gothic writers were quick to realize that Britain's growing empire could prove a vast source of frightening ,others' [...]." (Paravisini-Gebert 2002: 229)

$12 \mathrm{Vgl}$. zu Merleau-Pontys Bezügen auf Cézanne aber auch Paul Gauguin: Carbone (2011). 
karibische Philosoph und Schriftsteller Eduard Glissant mit seinen Überlegungen zur Créolité vorschlägt und damit allen „Ursubstanzen" eine Absage erteilt?

\section{Ausblick}

Wenn man so fragt, hat man es allerdings nicht nur mit einer kulturwissenschaftlichen Perspektive, sondern mit eminent philosophischen Angelegenheiten zu tun. Denn was wäre eine kritische Philosophie anderes als die Befragung der Voraussetzungen und Prämissen? Den Zusatz "kulturwissenschaftlich" braucht man vielleicht auch nur dort, wo die philosophische Disziplin der Ästhetik ihre eigenen Grundlagen nicht ausreichend ,provinzialisiert' und ihre über den Imperialismus hinausgehenden machtpolitischen Verflechtungen nicht konsequent genug befragt. Eine in diesem Sinn von den Cultural Studies her verstandene Ästhetik - und das ist die Perspektive für die Gegenwart, die wir eröffnen möchten - stellt ein kritisches Instrumentarium insbesondere in Bezug auf die okzidentale Tradition zur Verfügung.

Zur kulturwissenschaftlichen Provinzialisierung dieser Tradition der Ästhetik gehört es, die eigenen Grundbegriffe darauf hin zu befragen, welchen Verflechtungen sie sich verdanken und welche ästhetische Traditionen sie nicht fassen können. Dazu gehört aber auch die ästhetischen Begriffe zu pluralisieren, und zwar noch über die Intervention in die Geist-Körper-Dichotomie oder in die Hierarchie zwischen reinem und angewandtem bzw. nützlichem Schönen hinaus, wie dies schon längst im Rahmen dekolonialer Ästhetiken geschah und geschieht. Diese Öffnung gibt die philosophisch-kulturwissenschaftliche Ästhetik, für die wir plädieren, keinem schieren Relativismus preis. Gerade eine Ästhetik, die im Zwischenreich des Streits zwischen unterschiedlichen Ästhetiken operiert und keine für gegeben hält, wird sich darum bemühen, die eigenen Argumente und Vorurteile auf den Tisch zu legen. Damit kann sie das werden, was sie auch schon in der Vergangenheit punktuell war: ein potenzielles Residuum von self-othering und verflochtenen Allianzen. Dass sie im Effekt bisweilen regiozentrisch sein mag, liegt dann nicht am ,Westen' oder, globalen
Süden' per se. Solche Effekte sind vielmehr den jeweiligen Verflechtungen geschuldet, in die eine ästhetische Praxis nicht weniger als eine ästhetische Theorie durch ihre materielle und geopolitische Gebundenheit interveniert und die sie mikropolitisch mitkonfiguriert. Eine solche Perspektive ist nicht nur historisch zu sehen: Heute, um an Böhmes Bemerkungen zur Arbeit anzuschließen, betrifft es die viel diskutierten Fragen, inwieweit der globale Kapitalismus nicht nur durch die Ausbeutung von Körpern, sondern ebenso von Neigungen, Emotionen und Affektivitäten funktioniert und wie eine emanzipatorische ästhetische Praxis hier intervenieren kann. Eine den Cultural Studies verpflichtete Ästhetik wäre also eine solche, die erstens die Ästhetik(geschichte) auf ihre geo- und machtpolitischen Implikationen hin befragt, zweitens verflochtene Allianzen in den Vordergrund stellt und drittens im Sinne Glissants nach einer relationalen Ästhetik fragt. Ob der Zusatz "kulturwissenschaftlich" bei einer Hinterfragung der jeweiligen Gründungsfiguren einer Ästhetik und der Anerkennung der Pluralität der Kontexte und Bedingungen dann auch tatsächlich zu streichen wäre, hängt von der (Selbst-)Kritikfähigkeit der jeweiligen Ästhetik ab.

\section{Literaturverzeichnis}

Avermaete, Tom/Karakayali, Serhat/Osten, Marion von (Hgg.) (2010): Colonial Modern: Aesthetics of the Past, Rebellions for the Future. London: Black Dog Publishing.

Bernasconi, Robert (2001): Who Invented the Concept of Race? Kant's Role in the Enlightenment Construction of Race. In: Bernasconi, Robert (Hg.): Race. Malden/ MA: Blackwell, S. 11-36.

Bromley, Roger/Göttlich, Udo/Winter, Carsten et al. (Hgg.) (1991): Cultural Studies: Grundlagentexte zur Einführung. Lüneburg: zu Klampen Verlag.

Carbone, Mauro (2011): La chair des images: MerleauPonty entre peinture et cinema. Paris: Vrin.

Chakrabarty, Dipesh (2007): Provincializing Europe Postcolonial Thought and Historical Difference. Princeton: Princeton University Press.

Därmann, Iris (2005): Fremde Monde der Vernunft. Die ethnologische Provokation der Philosophie. München: Fink.

Därmann, Iris (2009): Landnahme, Menschennahme. John Locke und der transatlantische Sklavenhandel. In: Volker Gottowik et al. (Hgg.): Zwischen Aneignung und Verfremdung: ethnologische Gratwan- 
derungen. Festschrift für Karl-Heinz Kohl. Frankfurt a.M.: Campus, S. 69-82.

Diederichsen, Diedrich (1996): Nachsitzen für das ideale Institut. Von der Utopie des ganzheitlichen Gestalters zur Utopie von Cultural Producer und Cultural Studies. In: Spex 5, S. 53-55.

Ege, Moritz (2014): Tübingen/Birmingham: Empirische Kulturwissenschaft und Cultural Studies in den 1970er-Jahren. In: Historische Anthropologie 22/2, S. 149-181.

Engelmann, Jan (Hg.) (1999): Die kleinen Unterschiede. Der Cultural-Studies-Reader. Frankfurt a.M./New York: Campus.

Gikandi, Simon (2011): Slavery and the Culture of Taste. Princeton/Oxford: Princeton University Press.

Hall, Stuart (1980): Encoding/decoding. In: Centre for Contemporary Cultural Studies (Hg.): Culture, Media, Language: Working Papers in Cultural Studies, 1972-1979. London: Hutchinson.

Hall, Stuart et al. (1978): Policing the Crisis: Mugging, the State and Law and Order. London/Basingstroke: Macmillan Press.

Hall, Stuart/Evans, Jessica/Nixon, Sean (22013): Representation. Los Angeles et al.: Sage.

Hepp, Andreas (1999): Cultural Studies und Medienanalyse. Eine Einführung. Opladen/Wiesbaden: Westdeutscher Verlag.

Hörning, Karl H./Winter, Rainer (Hgg.) (1999): Widerspenstige Kulturen. Cultural Studies als Herausforderung. Frankfurt a.M.: Suhrkamp.

hooks, bell (2015): an aesthetic of blackness. In: bell hooks: yearning. race, gender, and cultural politics. New York: Routledge.

Kant, Immanuel (1974): Kritik der Urteilskraft (= Werkausgabe X). Hg. von Wilhelm Weischedel. Frankfurt a.M.: Suhrkamp.

Kant, Immanuel (1993): Beobachtungen über das Gefühl des Schönen und Erhabenen. Frankfurt a.M.: Anton Hain.

Kohl, Karl-Heinz (1981): Entzauberter Blick. Das Bild vom Guten Wilden und die Erfahrung der Zivilisation. Berlin: Medusa.
Leeb, Susanne (2015): Die Kunst der Anderen. ,Weltkunst' und die anthropologische Konfiguration der Moderne. Berlin: b_books.

Lettow, Susanne (2010): Philosophiegeschichte als Verflechtungsgeschichte. Globalität, Naturwissen und Kants Theorie der Menschenrassen. In: Zeitschrift für kritische Theorie 30-31, S. 26-46.

Mercer, Kobena (Hg.) (2005): Cosmopolitan Modernisms. Annotating Art's Histories: Cross-Cultural Perspectives in the Visual Arts. London: Institute of International Visual Arts and Cambridge, Mass.: MIT Press.

Mirzoeff, Nicolas (2011): The Right to Look. A Counterhistory of Visuality. Durham/London: Duke University Press.

Nelson, Cary/Treichler, Paula A./Grossberg, Lawrence (1992): Cultural Studies: An Introduction. In: Grossberg, Lawrence et al. (Hgg.): Cultural Studies. New York/London: Routledge.

Osten, Marion von/Maharaj, Sarat (2013): Der Überschuss des Globalen. Ein Gespräch zwischen Marion von Osten und Sarat Maharaj. In: Texte zur Kunst 91 (September), S. 133-152.

Paravisini-Gebert, Lizabeth (2002): Colonial and postcolonial Gothic: The Caribbean. In: Hogle, Jerrold E. (Hg.): The Cambridge Companion to Gothic Fiction. Cambridge: Cambridge University Press, S. 229-257.

Said, Edward W. (1993): Culture and Imperialism. New York: Alfred A. Knopf.

Smith, Woodruff (1991): Politics and the sciences of culture in Germany, 1840-1920. New York: Oxford University Press.

Sonderegger, Ruth (2004): Kunst als Sphäre der Kultur und die kulturwissenschaftliche Transformation der Ästhetik. In: Friedrich Jäger/Burkhard Liebsch/Jürgen Straub/Jörn Rüsen (Hgg.): Handbuch der Kulturwissenschaften. Bd. 3: Themen und Tendenzen. Stuttgart/Weimar: Metzler, S. 50-64.

Wuggenig, Ulf (2001): Neue Kulturwissenschaften an der Universität Lüneburg. In: Kulturpolitische Mitteilungen 94/II, S. 99-101. 